\title{
A HOLISTIC APPROACH TO THE SPACE DEBRIS MITIGATION
}

\author{
ALESSANDRO ROSSI (*) \\ Nota presentata dal s.c. Antonio Giorgilli \\ (Adunanza del 30 novembre 2017)
}

Sunto. - Le attività spaziali in quasi tutti i regimi orbitali sono ora a rischio a causa della continua crescita della popolazione dei detriti spaziali. Per prevenire la proliferazione dei detriti spaziali in orbita terrestre è necessario affrontare il problema da prospettive diverse, sfruttando le più avanzate conoscenze teoriche e sperimentali in campi diversi come l'astrodinamica, l'ingegneria aerospaziale e la giurisprudenza spaziale. Questo approccio permette di affrontare quattro pilastri principali: prevenzione, protezione, mitigazione e regolamentazione. A tale riguardo, la Comunità europea ha finanziato un progetto $\mathrm{H} 2020$ chiamato ReDSHIFT il cui obiettivo è trovare metodi passivi (ovvero che non richiedano uso di propellente aggiuntivo e l'intervento di veicoli spaziali ad hoc) per mitigare la proliferazione dei detriti spaziali.

$$
* * *
$$

Abstract. - The space activities in almost every orbital regime are now jeopardized by the continuous growth of the space debris populations. To prevent the proliferation of the space debris in Earth orbit it is necessary to tackle the problem from different perspectives, exploiting the latest theoretical and experimental knowledge in different fields, such as astrodynamics, spacecraft engineering and legal studies, to address four main pillars: prevention, protection, mitigation and regulation. In this respect the European Community financed a large $\mathrm{H} 2020$ project named ReDSHIFT whose goal is to find passive means to mitigate the proliferation of space debris. A short summary of the project and of its main findings is given in the paper, with particular emphasis on the more theoretical part, related to the simulation of long term evolutionary scenarios of the whole space debris environment and to the mapping of the Low Earth Orbit phase space, looking for passive dynamical de-orbiting solutions. 


\section{INTRODUCTION}

Our everyday life is increasingly relying on space. Telecommunications, global positioning, meteorological forecasts, disasters prevention and mitigation, safety and security are just few examples of the fields in which space is now a crucial resource. Nonetheless, the increasing number of space debris in Earth orbit is seriously jeopardizing the space assets in all the orbital regimes, from the very low orbits (at around $400-500 \mathrm{~km}$ of altitudes) up to the Geostationary region (at around $36000 \mathrm{~km}$ of altitude) where the TV, telecommunication and meteorological satellites are located.

The impact of debris on the space activities has to be reduced by adopting a global strategy able to address the problem from different points of view, from the very beginning of the planning of a space mission. As an example, the choice of the orbit, of the spacecraft bus, of the spacecraft power system and propulsion, are all aspects that influence, and have to be optimized, having in mind not only the goal of the mission but also the minimization of the "environmental" impact of the spacecraft. The space debris related aspects can be summarized in terms of: prevention, protection, mitigation and regulation. These aspects will be analyzed in the following by describing a large Horizon 2020 project, named ReDSHIFT, which is currently undergoing and is addressing all the above issues.

We note that, if none of the above measures proves to be effective enough, the remediation measures should be considered. This can imply the need to realize a dedicated space mission to actively remove hardware from space. The Active Debris Removal (ADR) solution is not part of the ReDSHIFT project and will not be discussed here. The interested reader can refer to, e.g., [3].

\section{The ReDSHIFT H2020 PROJECT}

In order to tackle the issues mentioned in the previous section, a large research project, called ReDSHIFT (Revolutionary Design of Spacecraft through Holistic Integration of Future Technologies) has been funded by the European Union in the framework of the PROTEC Call of Horizon 2020. The ReDSHIFT project is coordinated by the author, at IFACCNR (see http://redshift-h2020.eu/). In ReDSHIFT the debris mitigation goals will be achieved through a holistic approach that considers, 
from the outset, opposing and challenging constraints for the space environment preservation, the spacecraft survivability in the harsh space environment and the safety of humans on ground.

The main goal of ReDSHIFT is to tackle the space debris issue from a global perspective using expertises from several different fields: long term simulations of the space debris environment, astrodynamics, 3D printing, design for demise, protection and hypervelocity impact testing, legal and normative issues. The overall objective is to push for new, improved mitigation measures with the use of new theoretical results about the orbital dynamics of spacecraft coupled with new technologies, such as solar and drag sails (i.e., extremely light structures increasing the area of the spacecraft and exploiting either the gentle push of the solar radiation pressure -solar sail- or the drag produced by the thin residual atmosphere in case of low orbits, below about $800 \mathrm{~km}$ of altitude). The new paradigm of $3 \mathrm{D}$ printing, should be applied to enhance different aspects of the spacecraft design and manufacturing concurring to the mitigation efforts, such as, e.g., sail attachment and storage, shielding and design for demise.

The plot of the project is the following: first a through analysis of the currently adopted mitigation measures is performed to highlight their benefits and, possibly, their deficiencies in some aspects. This analysis is assisted by a number of simulations of the long term evolution of the space debris environment showing the overall effects of these measures in a measurable way. These simulations will serve also a the reference to assess the results achieved at the end of the project. This part will be described in Sec. 3.

While it is well known that the fundamental step to be performed to preserve the space environment is the disposal of the spacecraft at endof-life, sometime the maneuvers needed to achieve this goal are not practicable also for energetic or technical reasons. Therefore, a thorough understanding of the orbital dynamics in all the circumterrestrial space will allow us to identify stability and instability regions, and to exploit them to find preferential routes (we called them "de-orbiting highways") minimizing the energetic requirements for the operators, thus improving the applicability of the disposal maneuvers. Once identified, the best maneuvers needed to reach the "entrances" to the de-orbiting highways or the stable graveyard regions (in case the reentry option is not viable with the current technologies, e.g., for Geostationary satellites), coming from the used operational orbits, will be computed and the technical means to be 
used to accomplish those maneuvers will be identified. This part will be described in Sec. 4.

As a matter of fact, the project is focusing mainly on a few specific passive technologies (that is technologies not requiring propellant on board) such as solar and drag sails. The studies concerning the sails will be twofold: the theoretical aspects of the sail dynamics and the technological aspects of sail manufacturing will be both tackled. Moreover, since our main mitigation solution will be the de-orbiting towards the atmospheric reentry, the focus will also be on spacecraft designed for demise, that is a design that will minimize the chances that chunks of the spacecraft might reach the ground.

In an effort to make the above technological solutions easier and more attractive to produce and to implement into future spacecraft design, ReDSHIFT will explore the possibility to use the now blossoming additive manufacturing (3D printing) technology, to actually realize a model spacecraft and, in particular, some specific parts related to the debris mitigation issues, such as, e.g., the shielding, a sail canister, the sail hatches and joints, etc.

Once designed and realized, the prototypes will undergo specific test such as hypervelocity impacts, radiation, vibration, thermal testing, etc. This part will be briefly described in Sec. 5 .

In all the above process, a common thread linking highly theoretical analysis and more applied physics and engineering studies will be kept, in a constant attempt to stick to the proposed holistic vision.

At the end of the project the effectiveness of the proposed dynamical and technological solutions will be validated by repeating the long term simulations, and comparing the results with the ones obtained at the beginning of the project.

Beside the 3D parts, the final outputs of the projects will include also a software summarizing the theoretical and engineering findings, allowing the design of a space debris compliant mission (e.g., by suggesting the disposal trajectories and the technologies needed to achieve them, the best shielding opportunities for a given spacecraft and the possibility to produce it with additive manufacturing, etc.). It will output safe, scalable and cost-effective satellite and mission designs in response to operational constraints.

Last, but not least, all the experience gathered will be applied to the analysis of the normative related to space debris, in an effort to propose, to the proper forums new, improved mitigation practices and rules (Sec. 6). 


\section{LONG TERM SIMULATIONS OF THE SPACE DEBRIS ENVIRON- MENT}

The capability to model the long term evolution of the space debris environment is of paramount importance both to design the future missions and to test and assess the effectiveness of the proposed mitigation measures. In the last decades increasingly complex mathematical models were developed by the author's group, from the statistical one described in [10] to the semi-deterministic one, called Space Debris Mitigation (SDM) described in [11]. In these models, an initial population of objects present in space is evolved for time spans of the order of a few hundreds of years, integrating the orbits of every object and including all the source (e.g., launches, explosions, collisions, etc.) and sinks (e.g., natural perturbations such as air drag, active debris removal, mitigation measures, etc.) terms that are responsible for the evolution of the environment. Of particular importance is to be able to model the proposed mitigation measures, that is mainly operational practices aimed at reducing the orbital lifetime of spacecraft, once their mission is accomplished. In Low Earth Orbit (LEO, defined as the region of space from 0 to $2000 \mathrm{~km}$ of altitude) the main guideline is to dispose the spacecraft into an orbit whose residual lifetime, under the effects of the natural perturbations (atmospheric drag mainly), is not exceeding 25 year. This is the so-called 25-year rule widely adopted by the space agencies worldwide as a pillar of the mitigation strategies aimed at protecting the LEO zone.

To assess and quantify the effectiveness of the most common measures, specific long term debris environment simulations were performed with SDM (and with a similar software developed by the Technical University of Braunschweig, called LUCA [9]). In particular, the simulations included an analysis of the effects of the level of compliance to the mitigation measures (i.e., how many missions actually respect the proposed guidelines - see Sec.6), of the residual lifetime of the spacecraft at their end-of-life (25 year with respect to 10 years), of the collision avoidance procedures, of the active debris removal and of the proposed launch in space of very large constellations of satellites in Low Earth orbit [8].

As an example, the left panel of Fig. 1 shows the comparison of the effective number of objects in LEO for three simulated scenarios, over a time span of 200 years. The blue line is the "Reference" case and it depicts a scenario where the space operations are supposed to be performed in a way similar to the one currently adopted. In particular it is assumed that the spacecraft are de-orbited at the end-of-life respecting the 25-year rule, 
but only with a $60 \%$ compliance level. The magenta curve is based on the same Reference scenario, but it assumes the use of Active Debris Removal of two objects per year. In particular, every year, the two most dangerous objects (i.e., the objects having the highest product between their mass and their collision probability against the other objects in space) are removed from the simulation. Finally, the red line refers to a scenario like the Reference one, but the spacecraft are moved at the end-of-life to an orbit with only 10 years of residual lifetime and, moreover, a $90 \%$ compliance to this 10-year rule is simulated. The right panel of Fig. 1 shows the cumulative number of catastrophic collisions happening in the simulated time span for the three scenarios (with the same color code). A few comments to Fig. 1 are in order:

- the improved compliance to the currently proposed mitigation mea sures gives the best results in terms of the growth of the LEO population and of the expected catastrophic collisions;

- notwithstanding the above mentioned improvement, the environment is still "unstable" and we observe a constant growth of the number of objects in LEO;

- the active removal of just two objects per year is not significantly improving the situation, but still produces a decrease of about $12 \%$ of the final population. It is worth mentioning here that the technology for ADR is now almost mature and about to be tested. On the other hand the political and economic issues related to future ADR missions are still to be overcome.

The results of the simulations give the expected quantitative evaluation of the effectiveness of the different measures and represent the reference against which to compare the final results of the ReDSHIFT project.

Whereas the full details of the simulation results can be found in [13], here it is important to raise a warning that is evident looking at Fig. 2.

The Figure shows the spatial density of objects in LEO for the Reference and the improved mitigation scenarios for the epoch 2213 (end of the simulated time span) as a function of altitude, compared with the initial epoch. A general improvement of the situation can be noted, especially in the crowded critical regions around 900 and $1400 \mathrm{~km}$, due to the increased number of satellites de-orbited at the end-of-life. Nonetheless, looking carefully at the higher end of the plot, around $2000 \mathrm{~km}$ of 

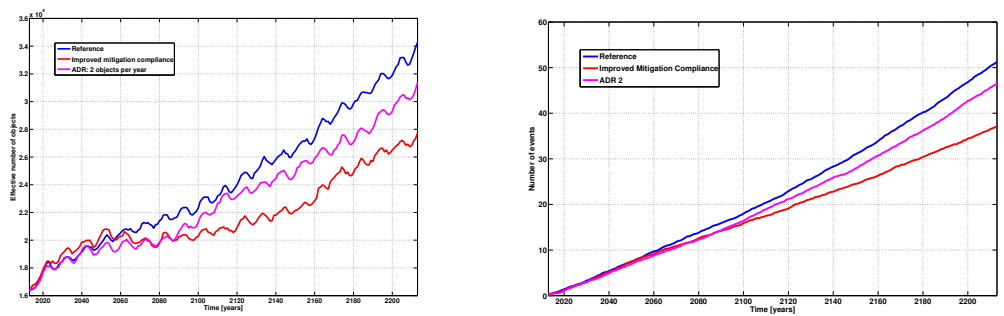

Fig. 1 - Effective number of objects (left panel) and cumulative number of fragmentations (right panel), in LEO, for three different evolutive scenarios. See text for details

altitude, an opposite behavior, with a growth of the density in the mitigation scenario, can be noticed. This is due to the following reason: for high LEO orbits (above about $1400 \mathrm{~km}$ of altitude) whenever an object has to be disposed at the end-of-life, the best solution, in terms of the $\Delta V$ required for de-orbiting, might be not the usual de-orbiting to a lower elliptical orbit with a defined residual lifetime. Instead, a re-orbiting into a circular "graveyard" orbit above the LEO protected zone (i.e., above 2000 $\mathrm{km}$ of altitude) can be energetically more convenient. Hence, a number of objects is usually moved, to this kind of graveyard. Therefore the mathematical model is able to choose the optimal solution between the two options (in terms of the required $\Delta V$ ). In the plot of Fig. 2 we are starting to see not only the simple effect of the accumulation of the disposed satellites in the super-LEO graveyard, but also the growth of fragments generated by the mutual collisions between the uncontrolled disposed spacecraft (unable to perform avoidance maneuvers).

This result reinforces the conclusion that novel disposal means should be devised also to lower the energetic requests of de-orbiting maneuvers, thus minimizing the recourse to the LEO graveyard zones. The mapping of the phase space described in the next Section, with the search for the "de-orbiting highways" is devoted exactly to this purpose.

\section{DYNAMICAL MAPPING OF THE CIRCUMTERRESTRIAL SPACE}

As shown in the previous section, the disposal of objects outside the LEO protected region is one of the most important mitigation measure needed to reduce the growth of space debris in the forthcoming years. In order to 


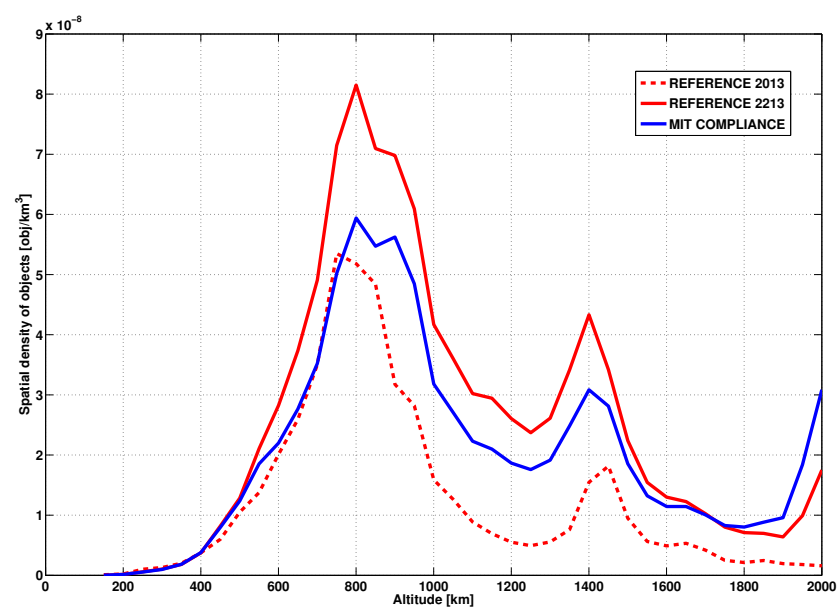

Fig. 2 - Spatial density of objects (objects $/ \mathrm{km}^{3}$ ) larger than $10 \mathrm{~cm}$ in LEO for the Reference (red line) and improved mitigation compliance cases (blue line) in the year 2213. The dashed red line represents the initial density at epoch 2013.

facilitate this action it is important to identify stable and unstable regions in the phase space where the objects could be moved to exploit either long term "graveyards" or, possibly and preferentially, faster escape routes. To this purpose, a dynamical mapping of the circumterrestrial space was performed within ReDSHIFT, including the whole space from LEO up to the geostationary orbit.

The circumterrestrial space was divided in a fine grid in the orbital elements space and about 20 millions of initial conditions were propagated, with three different orbital propagators, having similar dynamical models expressly tailored to the explored orbital regime, but different underling computational engines. All the orbital propagations were repeated twice: once assuming for the test object an Area-to mass ratio equal to the standard satellites $\left(0.012 \mathrm{~m}^{2} / \mathrm{kg}\right)$ and then assuming an augmented area $\left(1 \mathrm{~m}^{2} / \mathrm{kg}\right.$, thus simulating the presence of some kind of sail). The output of these massive orbital propagations were stored in terms of maps displaying, e.g., the lifetime or the maximum eccentricity growth for all the orbits during the 120 years time span of the propagation.

Here a short summary of the results for the LEO region will be pre- 
sented. For more information on the mapping of the higher orbits the reader can refer to [14] and [5].

In LEO the main purpose of the dynamical disposal studies is to identify natural perturbations able to increase the orbital eccentricity in order to bring the spacecraft to interact with the atmosphere at perigee, hence shortening its lifetime. As a first step, it is worth identifying the orbits from which an abandoned object can "naturally" reenter, within a given time span, without any additional maneuver. In Fig. 3, for typical intact objects having a ratio between area and mass $A / m=0.012$ $\mathrm{m}^{2} / \mathrm{kg}$, the minimum value of eccentricity $(e)$, as a function of the semimajor axis, which guarantees a lifetime of at most 25 years is shown (blue line in the Figure). The red line shows instead the minimum value of eccentricity, for any value of inclination in the range explored and for each given semi-major axis, which ensures to reenter within the total time span of our exploration (120 years). Finally, the cyan stars represent cases where perturbations, different from drag, facilitate the reentry at specific values of inclination (see later).

Building on the results shown in Fig. 3, the LEO space is explored highlighting a strong network of dynamical resonances responsible for the growth of the orbital eccentricity able to improve the picture shown in the blue and red lines of Fig. 3, further shortening the residual lifetime. At difference from the dynamics of the higher orbits in the medium Earth Orbit region (MEO, at altitudes around $15000-20000 \mathrm{~km}$ ), in LEO the perturbing effects can be obtained mostly not from lunisolar resonances, but also from high-degree zonal harmonics and solar radiation pressure.

The complete mapping of the LEO space, over the selected grid of initial conditions, produced a large number of phase diagrams such as the ones shown in Fig. 4.

The two panels show, as a function of initial inclination and eccentricity, the maximum eccentricity reached (see color bar) over the 120-year time span by an object with initial semimajor axis $a=R_{\oplus}+1560 \mathrm{~km}$, where $R_{\oplus}$ is the radius of the Earth (initial right ascension of the ascending node $\Omega=0$ and argument of perigee $\omega=0^{\circ}$ ). The left panel refers to an object having $A / m=0.024 \mathrm{~m}^{2} / \mathrm{kg}$ and the right panel to an object having $A / m=1 \mathrm{~m}^{2} / \mathrm{kg}$. A few things can be noted from this example. First the enhanced effects due to the increase in the $A / m$ of the object is apparent. Then, the bright "corridors" that are visible mainly in the right panel, clearly show how, at specific inclinations (e.g., at $i \sim 40^{\circ}$ or $i \sim 80^{\circ}$ ) the perturbing effects are particularly enhanced. These corri- 


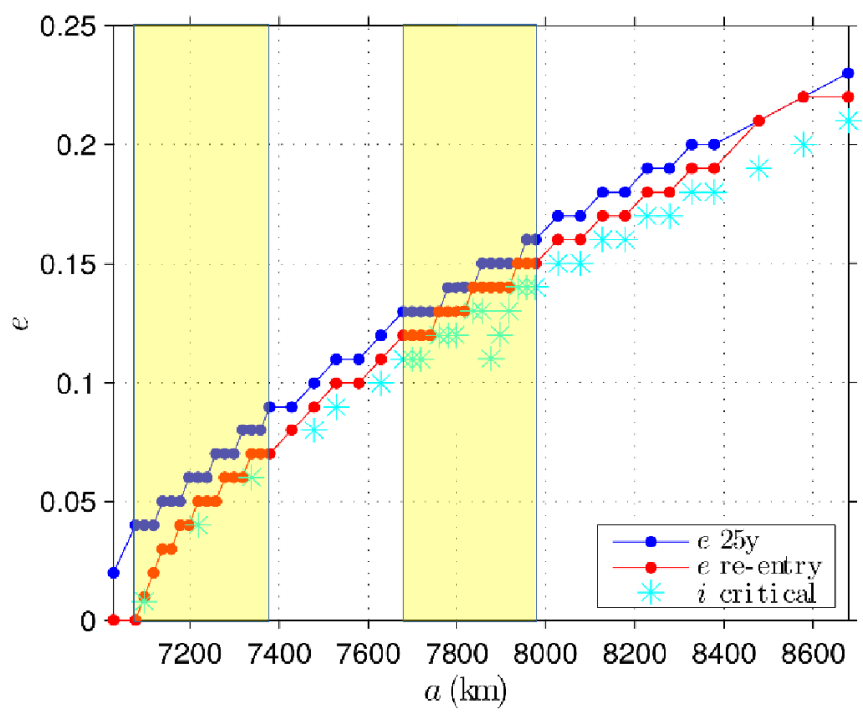

Fig. 3 - As a function of the semi-major axis, the minimum value of eccentricity required to reenter in 25 years (blue curve), and in less than 120 years (red curve), assuming $A / m=0.012 \mathrm{~m}^{2} / \mathrm{kg}$, and $\Omega=180^{\circ}$ and $\omega=0^{\circ}$ and the initial epoch 2020. The cyan stars represent cases where perturbations different from drag facilitate the reentry at specific values of inclination (see text for details). In yellow, the most populated altitudes are bighlighted.

dors correspond to resonant inclinations, associated with the cyan stars shown in Fig. 3. At these specific inclinations, the perturbation exerted by the geopotential or by the Moon or by the Sun (both gravitational or as solar radiation pressure) are able to alter the general behavior.

A specific analysis of these resonances was performed. All the details can be found in [2] and [1]. Here the main findings are briefly summarized. In Figure 5 and Figure 6 (top panel), we show, the maximum value, numerically computed, for the eccentricity in 120 years as a function of the initial inclination and semi-major axis, for prograde orbits starting from $e=0.02, \Omega=0^{\circ}, \omega=0^{\circ}$, for spacecraft having $A / m=0.012$ $\mathrm{m}^{2} / \mathrm{kg}$ and $A / m=1 \mathrm{~m}^{2} / \mathrm{kg}$, respectively.

In Fig. 5 the maximum value, numerically computed, for the eccentricity in 120 years as a function of the initial inclination and semi-major 

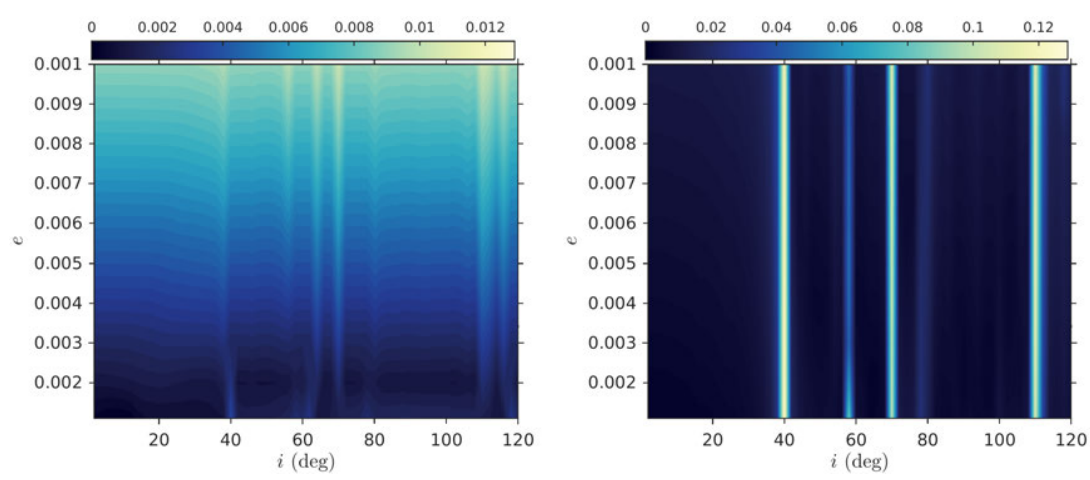

Fig. 4-Maximum eccentricity computed over 120 years (color bar) for quasi-circular orbits as a function of initial inclination and eccentricity for $a=R_{\oplus}+1560 \mathrm{~km}$, $\Omega=90^{\circ}, \omega=0^{\circ}$ at the initial epoch 2020. Left: $C_{R}(A / m)=0.012 \mathrm{~m}^{2} / \mathrm{kg}$; right: $C_{R}(A / m)=1 \mathrm{~m}^{2} / \mathrm{kg}\left(C_{R}\right.$ is the reflectivity coefficient varying between 1 and 2$)$. Note the change of scale in the color bars between the left and the right panels.

axis, for prograde orbits starting from $e=0.02, \Omega=0^{\circ}, \omega=0^{\circ}$ for a spacecraft with $\mathrm{A} / \mathrm{m}=0.012 \mathrm{~m}^{2} / \mathrm{kg}$. Figure 6 shows the same quantities for a spacecraft with $\mathrm{A} / \mathrm{m}=1 \mathrm{~m}^{2} / \mathrm{kg}$. The yellow lines identify the locations of the following resonances:

- $\quad$ singly-averaged solar gravitational resonances (e.g., [7])

$$
\begin{aligned}
& -\dot{\psi}=2 \dot{\omega}+\dot{\Omega}-2 n_{S} \approx 0, \\
& -\dot{\psi}=2 \dot{\omega}-n_{S} \approx 0, \\
& -\dot{\psi}=2 \dot{\omega}+2 \dot{\Omega}+n_{S} \approx 0, \\
& -\dot{\psi}=2 \dot{\omega}+2 \dot{\Omega} \approx 0
\end{aligned}
$$

where $\psi$ is the critical angle of the resonance, $n_{S}$ is the apparent mean motion of the Sun and $\dot{\Omega}$ and $\dot{\omega}$ are the time derivatives of the right ascension of the ascending node and of the argument of perigee, respectively;

- $\dot{\psi}=2 \dot{\omega}+\dot{\Omega} \approx 0$, associated with doubly-averaged lunisolar gravitational perturbations (e.g., [7]); 


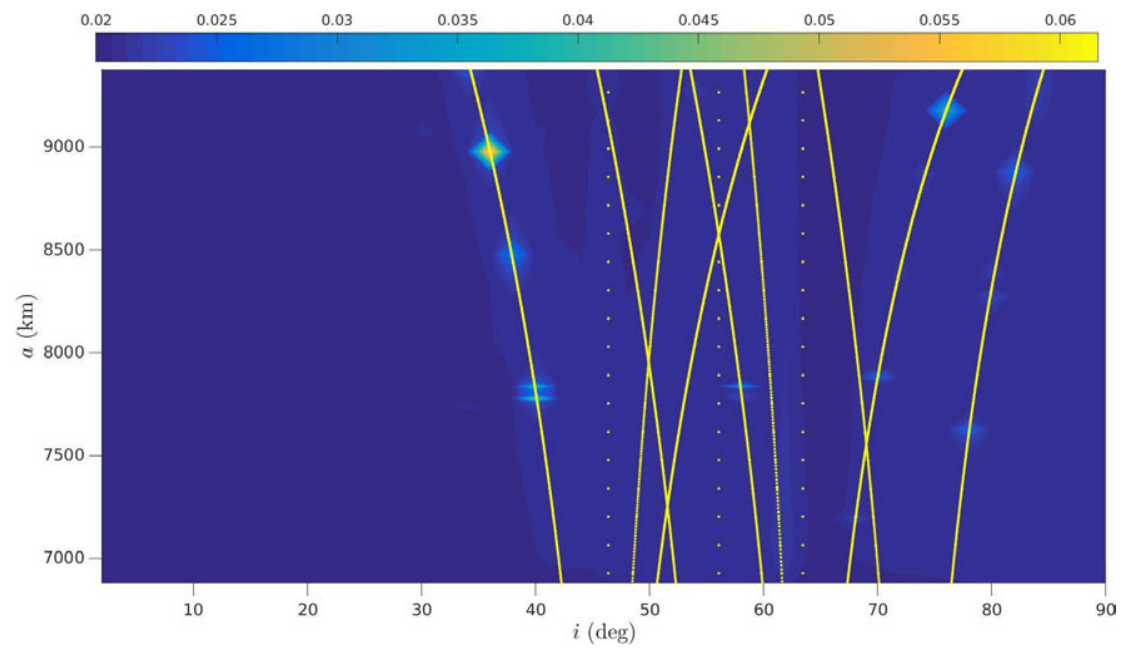

Fig. 5-As a function of the initial inclination and semi-major axis, the maximum eccentricity value (color bar) computed in 120 years starting from $e=0.02, \Omega=$ $0^{\circ}, \omega=0^{\circ}$, for the initial epoch 2018, considering $C_{R} A / m=0.12 \mathrm{~m}^{2} / \mathrm{kg}$ and prograde orbits. The yellow lines denote the dominant resonances determining the longterm behavior.

- $\dot{\psi}=\dot{\omega} \approx 0$, associated with lunisolar perturbations (e.g., [7]), but also with high-degree terms in the geopotential (e.g., [12]);

- $\dot{\psi}=\alpha \dot{\Omega} \pm \dot{\omega} \pm n_{S} \approx 0$, associated with SRP $(\alpha=0,1)$, [4]-[6].

For $A / m=1 \mathrm{~m}^{2} / \mathrm{kg}$, the only resonances which matter are the six resonances associated with SRP, namely those in the last bullet, which can be rewritten, for the sake of clarity as,

$$
\begin{aligned}
& \dot{\psi}_{1}=\dot{\Omega}+\dot{\omega}-n_{S} \approx 0, \\
& \dot{\psi}_{2}=\dot{\Omega}-\dot{\omega}-n_{S} \approx 0, \\
& \dot{\psi}_{3}=\dot{\omega}-n_{S} \approx 0, \\
& \dot{\psi}_{4}=\dot{\omega}+n_{S} \approx 0, \\
& \dot{\psi}_{5}=\dot{\Omega}+\dot{\omega}+n_{S} \approx 0, \\
& \dot{\psi}_{6}=\dot{\Omega}-\dot{\omega}+n_{S} \approx 0 .
\end{aligned}
$$



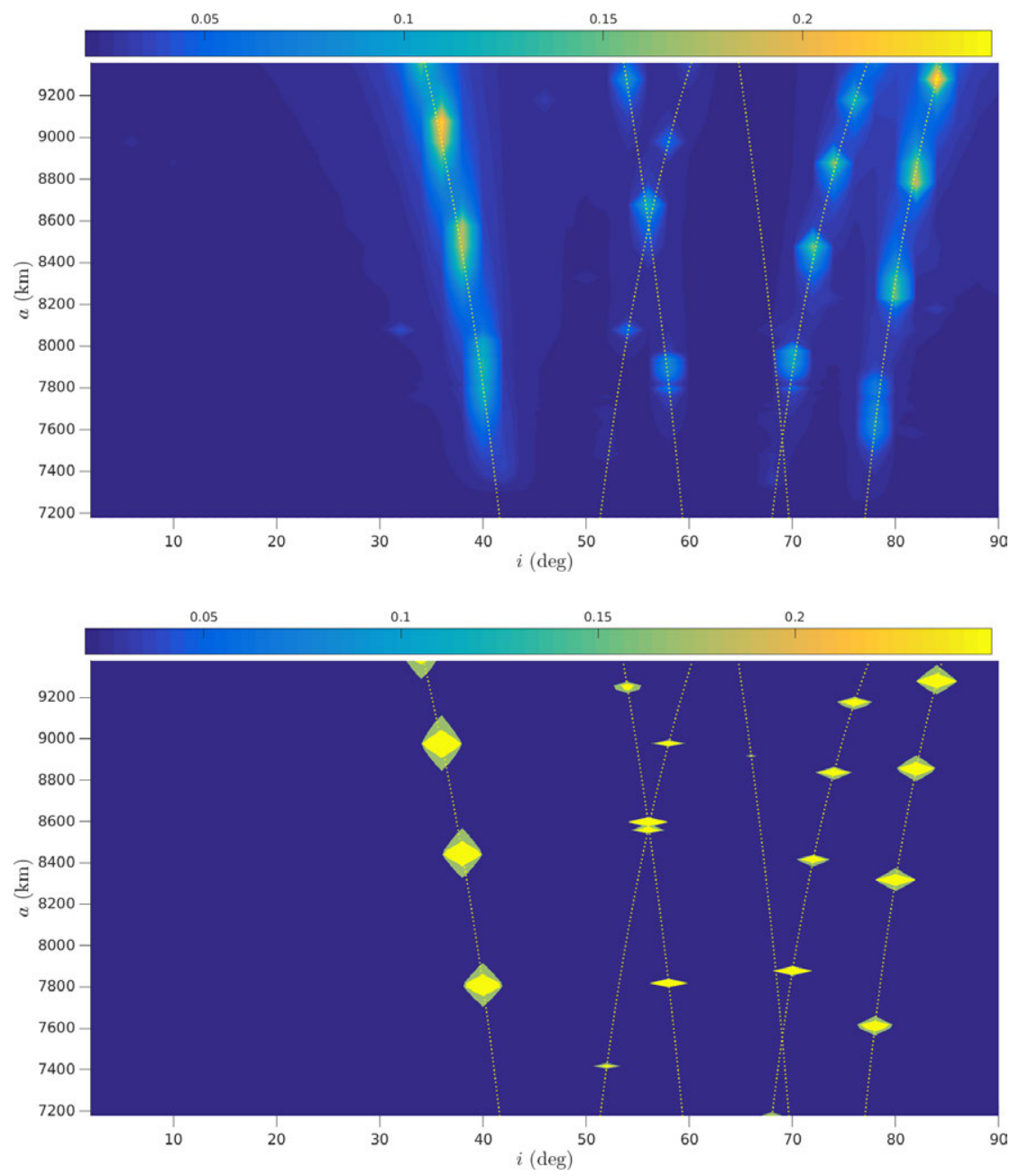

Fig. 6 - Top panel: as a function of the initial inclination and semi-major axis, the maximum eccentricity value (color bar) computed in 120 years starting from $e=0.02$, $\Omega=0^{\circ}, \omega=0^{\circ}$. for the initial epoch 2020, considering $C_{R} A / m=1 \mathrm{~m}^{2} / \mathrm{kg}$ and prograde orbits. Bottom panel: analytical estimate of the maximum variation in eccentricity is shown. The yellow lines denote the dominant resonances determining the long-term behavior. 
From Figure 6 (top), it can be noted that, if an area augmentation device able to increase the $A / M$ ratio is attached to the spacecraft, the variation in eccentricity that can be obtained at a resonance can definitely allow for a reentry. As a matter of fact, it turns out that the amount of eccentricity increase can be estimated analytically. The derivation is not presented here, but the reader can refer to [2] and [1]. The bottom panel of Fig. 6 shows the location of the resonances computed analytically; the good correspondence with the numerical results of the top panel can be noted.

All the maps, similar to those of Fig. 4, for the whole LEO to GEO space, are now stored and will be assembled in an "atlas" which will be publicly available on the ReDSHIFT web site and will be used to identify, for every orbital regime the most convenient de-orbiting or re-orbiting options. Moreover the computation of the optimal maneuvers needed to steer, whenever possible, the spacecraft towards the resonances at the end-of-life are being computed.

\section{DESIGN, 3D PRINTING AND TESTING OF NOVEL SPACECRAF:}

As mentioned in Sec. 1, there is the need to tackle the space debris problem from different points of view. The design and building of the spacecraft has to be defined from the very beginning taking into account the harsh environment where the satellite will operate.

It is important to design satellites that can survive impacts against small particles (below $\sim 1 \mathrm{~cm}$ in size) in order to minimize the in orbit failure that, not only compromise the mission objectives, but also leave a stranded spacecraft in the operational orbits, preventing any disposal maneuvers. This can be accomplished by protecting the satellite, or specific vital section of it, with ad-hoc light-weight shields, with different materials. Whenever the shielding is not possible (i.e., for larger particles), it would be desirable to have a spacecraft which could possibly minimize the production of small debris after a fragmentation. This could be accomplished by designing the satellite with, e.g., pre-defined breakup lines bounded to fail in case of a large impact and less prone to transport the shock to the whole structure.

These complex engineering aspects are dealt with in ReDSHIFT. By using 3D printing, it is possible to test different designs and materials in a fast and relatively economical way. Multiple spacecraft parts can be printed, by changing some details, and then tested in specific facilities such as impact laboratories with light gas guns (to perform hypervelocity 
impacts tests), vibration and radiation chambers, etc. Addictive manufacturing at component level will include also multifunctional structures testing novel solutions like embedded wiring (structronics), power generation, electronic shielding, heat dissipation,..... Moreover, as mentioned in the previous section, the possibility to add to a spacecraft, from the design phase, as de-orbiting device (such as a sail) can represent a fundamental tool to achieve a fast de-orbiting. Once again, 3D printing will allow the testing of novel interfaces between de-orbiting devices and the satellite body thus enhancing the applicability of these kind of solutions.

A parallel research activity is devoted to the so-called "design-fordemise" (D4D). While de-orbiting into the atmosphere is an ideal solution to prevent the proliferation of space debris, it is important to avoid the accidental fall of material on inhabited areas. Whenever a controlled re-entry of the spacecraft, targeting oceans or deserts, is not possible, it has to be checked that no spacecraft parts can survive the atmospheric heating and reach the ground. That is, the spacecraft has to be designed to demise into the atmosphere. This implies a number of preliminary theoretical studies to identify the re-entry trajectories that guaranty the highest heat load on the spacecraft structures that, in turns, have to be designed in such a way to facilitate the disruption during the re-entry. This can be a requirement conflicting with the above mentioned need for impact resistance and it is therefore a crucial design task to be defined at the very beginning of the spacecraft production. Every material used and every larger test part printed during the project will be therefore also tested for demise in an arc-heated hypersonic wind tunnel.

It is worth stressing again that, trying to keep the holistic approach as in the title of the project, all the design activities have to be performed in close collaboration with the theoretical findings described in the previous sections. E.g., the design and production have to take into account the debris environment expected during the mission lifetime, as derived from the long term simulations described in Sec. 3, to define the needed level of shielding and protection. Moreover, the de-orbiting options and trajectories, found for the specific orbital region, have to be considered in order to design the best de-orbiting devices able to steer the spacecraft towards the most favorable "de-orbiting highway" at the end-of-life. This interaction between the different aspects of the project will be engineered with the production of a software suite (made publicly available through the project web site) providing a complete debris mitigation analysis of a mission. 
This phase of the project is now ongoing. The first detailed designs of the test spacecraft, shields and sail interface were produced. The first printed samples and tests shall start in the coming months.

\section{LEGAL AND POLITICAL ASPECTS OF THE SPACE DEBRIS MIT- IGATION}

As mentioned in Sec. 3, the potential benefits of any proposed mitigation measure is often weakened by its limited practical application. A clear understanding of the complex legal framework of the space activities is therefore essential to try and enforce any innovative mitigation procedure. The ReDSHIFT project will address also this aspect in an effort to maximize the effectiveness of its technical findings.

Currently, the enforcement of the existing mitigation rules, such as, e.g., the IADC (Inter Agency Space Debris Coordination Committee) and the UNOOSA ((Unites Nation Office for Outer Space) guidelines, is complicated by the fact that the guidelines are not legally binding (i.e., there are no legal consequences if they are not followed) but configure themselves as voluntary recommendations. That is, from a strictly legal perspective, it is the legal character of the existing mitigation rules that prevents their enforcement. i.e., the guidelines are explicitly dependent on voluntary compliance, hence no enforcement mechanism is foreseen. From an economic point of view, there are mostly cost-related and economic competition considerations which prevent from applying the guidelines as recommended. As the mitigation guidelines are, as mentioned above, not legally binding some other motive might be needed to stimulate their observance. "Steering" instruments comparable to the emissions trading in environmental policies could be the so-called "steering taxes", even if the application of this option is extremely delicate and of very difficult predictability with respect to their possible effects, as the space is a global market and a rather exclusive one due to the high costs involved. Other means could be state subsidies aiming at stimulating private actors to comply with mitigation standards. In the course of ReDSHIFT investigations in these direction will be performed.

Otherwise the guidelines have to be transformed to legally binding rules of international law. Mandatory legal character could be attributed to the guidelines also by virtue of national laws which foresee adherence to the standards contained in the guidelines. Individually some European countries have undertaken legal actions to deal with the space debris. Ex- 
amples include the United Kingdom's Outer Space Act, German efforts at regulation, the French Space Act, the draft European Code of Conduct 2004, and the ESA Space Debris Mitigation Guidelines of 2008. In some countries, licenses can be issued only if operations are conducted in such a way to prevent the contamination of outer space with debris. All these national legislations are being analysed, together with the international guidelines, to identify benefits and flaws and to find common grounds between them trying to push Europe to act as a single entity to promote regulations on the global political forums

Since an object abandoned in space represents a risk for any operational satellite, another aspect of the problem is the liability in case a damaging collision would take place. According to the Liability Convention, a launching State remains the "owner" of a space object even after it has come to the end of its lifespan and has become an orbital debris. However, there is no concrete provision for a duty to remove debris in international law and the "legal" identification of the origin of small space debris (e.g., fragments) is practically unfeasible and the attribution of fault for liability is therefore actually impossible. The development of an insurance market for space projects would be a very important prerequisite for the practicability and applicability of space debris remediation and mitigation rules. Though technically and economically problematic innovative solutions could be hypothesized. E.g., the establishment of an insurance fund where all space actors pay a certain amount which is used for space environment remediation or for the consequences of space debris accidents.

In the analysis of an optimised legal and political framework able to better promote the application of improved guidelines it is important to explore how the new design and technologies and rules foster specific branches of the economy. In the environmental economy there are clear evidences that, contrary to common feelings and initial expectations, new rules are often fostering innovation urging manufacturers to move from the status quo towards new, often economically profitable technical solutions. The aim of the legal/political and economical analysis within ReDSHIFT is also to push the space manufacturers towards these innovative and possibly profitable directions. 


\section{Conclusions}

To prevent the proliferation of the space debris in Earth orbit it is necessary to tackle the problem from different perspectives, exploiting the latest theoretical and experimental knowledge in different fields such as astrodynamics, spacecraft engineering, legal studies. The H2020 ReDSHIFT project was devised to answer to these needs. In particular in this note the main results reached up to now, especially in the dynamical mapping of the LEO region were described. A number of "corridors" related to specific resonances between gravitational and non-gravitational perturbation effects were identified. These resonances can be exploited to facilitate the de-orbiting of the spacecraft at the end-of-life by increasing the orbital eccentricity. The experimental part of the ReDSHIFT project has just started and the final results are expected by the end of the year 2018 .

Acknowledgements. The research leading to these results has received funding from the Horizon 2020 European Union's Framework Programme for Research and Innovation (H2020-PROTEC-2015) under REA grant agreement n. [687500]-ReDSHIFT. The work presented here was performed by the ReDSHIFT team whose contribution is gratefully acknowledged. In particular E.M. Alessi and G. Schettino were responsible for most of the work described in Sec. 4

\section{REFERENCES}

[1] Alessi E.M., G. Schettino, A. Rossi, G.B. Valsecchi Solar Radiation Pressure Resonances in Low Earth Orbits, MNRAS, in press, 2017.

[2] Alessi E.M., G. Schettino, A. Rossi, G.B. Valsecchi Natural Highways for End-ofLife Solutions in the LEO Region, CeMDA, submitted, 2017.

[3] Bombardelli C., E.M. Alessi, A.Rossi, G.B. Valsecchi, Environmental effect of space debris repositioning, Advances in Space Research, 60, 28-37, 2017.

[4] Cook, G. E., Luni-solar perturbations of the orbit of an Earth satellite, The Geophysical Journal of the Royal Astronomical Society, 6, 271-291, 1962.

[5] Colombo C. and Gkolias I., Analysis of orbit stability in the Geosynchronous region for end-of-life disposal, Proceedings of the 7th European Conference on Space Debris, Darmstadt, Germany, 2017.

[6] Hughes, S., Satellite orbits perturbed by direct solar radiation pressure: general expan- sion of the disturbing function, Planetary and Space Science, 25, 809-815, 1977. 
[7] Hughes, S., Earth Satellite Orbits with Resonant Lunisolar Perturbations. I. Resonances Dependent Only on Inclination, Proceedings of the Royal Society of London. Series A, Mathematical and Physical Sciences, 372, 243-264, 1980.

[8] Lewis H., J. Radtke, A. Rossi et al., Sensitivity of the Space Debris Environment to Large Constellations and Small Satellites,JBIS, 70, 105-107, 2017.

[9] Radtke J., S. Muller, V. Schaus, and E. Stoll, LUCA2 - an enhanced long-term utility for collision analy sis, in Proceedings of the 7th European Conference on Space Debris, Darmstadt, Germany, 2017.

[10] Rossi A., Cordelli A., Farinella P., Anselmo L., Collisional evolution of the Earth's orbital debris cloud, Journal of Geophysical Research, 99, 23195-23210, 1994.

[11] Rossi A., L. Anselmo, C. Pardini, R. Jehn, and G. B. Valsecchi, The new Space Debris Mitigation (SDM 4.0) long term evolution code, in Proceedings of the Fifth European Conference on Space Debris, Darmastadt, Germany, 2009.

[12] Roy, A. E., Orbital Motion, Institute of Physics Publishing, Bristol, 2005.

[13] Schaus V., J. Radtke, E. Stoll, A. Rossi, C. Colombo, S. Tonetti, I. Holbrough, Results of reference long-term simulations focussing on passive means to reduce the impact of space debris, in Proceedings of the 7th European Conference on Space Debris, Darmstadt, Germany, 2017.

[14] Skoulidou, A. J. Rosengren, K. Tsiganis, and G. Voyatzis, Cartographic study of the MEO phase space for passive debris removal, in Proceedings of the 7th European Conference on Space Debris, Darmstadt, Germany, 2017. 
